\title{
Character Association and Path Co-efficient Analysis for Yield Attributing Traits in Dahlia (Dahlia variabilis L.)
}

\author{
Maguluri Sree Devi*, G. K. Seetharamu, B. C. Patil, C. N. Hanchinamani, \\ Laxman Kukanoor, D. Satish and Sandhyarani Nishani
}

Kittur Rani Channamma College of Horticulture, Arabhavi, University of Horticultural
Sciences, Bagalkot, Karnataka, India

*Corresponding author

\author{
A B S T R A C T
}

\begin{tabular}{|l|}
\hline Ke y w o r d s \\
$\begin{array}{l}\text { Dahlia, } \\
\text { Correlation, Path } \\
\text { analysis, Selection }\end{array}$ \\
\hline Article Info \\
\hline $\begin{array}{l}\text { Accepted: } \\
\text { 24 July 2020 } \\
\text { Available Online: } \\
\text { 10 August } 2020\end{array}$ \\
\hline
\end{tabular}

\section{Introduction}

Dahlia (Dahlia variabilis L.) is a tuberous rooted herbaceous perennial belonging to the family Asteraceae having its origin in Mexico. It is popular plant for landscaping, cut flower and loose flower purposes (Smith, 1971).Knowledge on inter-relationship of characteristics of crop is of paramount importance as it helps in selecting appropriate components, which would result with improvement of complex characteristics that are correlated with each other (Al-Jibourie et al., 1958). However, ccorrelation coefficient alone cannot provide a complete representation of the causal basis of relationship and path coefficient analysis is relied upon to do so (Islam and Khan, 1991 and McGiffen et al., 1994). Therefore, the present investigation was undertaken to estimate associations among desired traits and their direct and indirect contributions toward yield in thirty two cultivars of dahlia.

\section{Materials and Methods}

The experiment was carried out at department of Floriculture and Landscape Architecture, Kittur Rani Channamma College of Horticulture, Arabhavi which is situated in the 
Northern dry zone (Zone III) of Karnataka. The experiment was laid out in Randomized Block Design with spacing of $60 \mathrm{~cm} \times 40 \mathrm{~cm}$, which was replicated twice with 32 genotypes in open field condition. Treatments details of cultivars used are enlisted in Table 1.Recommended agro techniques were followed and observations were made on the different vegetative and floral parameters. Genotypic and phenotypic correlation coefficients were calculated according to the formula suggested by Johnson et al., (1955) and Hanson et al., (1956). Correlation coefficient were further partitioned into components of direct and indirect effects by path coefficient analysis originally developed by Wright (1921) and later described by Dewey and Lu (1959).

\section{Results and Discussion}

Yield is a complex trait determined by several other parameters. Hence, the association of these characters with yield and among themselves is of paramount factor in selection of best genotypes. It is evident from Table 2 that, flower yield per plant had a highly significant positive genotypic correlation with leaf area index (0.617), crop duration (0.771), flowering duration (0.800), tuber weight (0.668) and change in fresh weight at day 3 (0.347), while non-significant negative correlation was observed between flower yield per plant and plant height at 90 DAP (0.021). A positive non-significant association with flower yield per plant was observed for all the other traits. A highly significant positive phenotypic correlation was observed between flower yield per plant and leaf area index (0.592), duration of crop (0.686) and duration of flowering (0.778), while tuber weight (0.646) showed a significant positive correlation. All other traits except plant height at 90 DAP showed a non-significant positive correlation (Table 2). These observations regarding vase life were in parallel with studies done by Mathad et al., (2005) in marigold; Kumari et al., (2017) in chrysanthemum. The degree of association between characters as indicated by the correlation coefficients has always been a helpful instrument for the selection of desirable characters under a breeding program (Islam et al., 2010).

According to Table3, at genotypic level, duration of crop (5.848), duration of flowering (2.663) and flower diameter (2.506) had a very high direct positive effect on flower yield per plant while vase length (0.770), total chlorophyll content $(0.595)$ and change in fresh weight at day $3(0.419)$ had a high direct positive effect. Plant height at 90 DAP showed a negligible positive effect whereas, water uptake at day 3 (-2.390), plant spread in E-W (-1.664), stalk length $(-1.593)$, tuber weight $(-1.508)$ and LAI (-0.373) showed a direct negative. plant height at 90 DAP had a non-significant negative correlation with flower yield per plant (-0.021) due to indirect negative effect via water uptake at day 3 (-1.475), plant spread in E-W (-1.210), stalk length (-1.119), total chlorophyll content (-0.204) and duration of flowering (-0.003) whereas, flower diameter (2.095), duration of crop (1.309), vase life (0.376), change in fresh weight at day $3(0.081)$, LAI (0.045) and tuber weight (0.029) had an indirect positive effect. duration of crop had a positive and highly significant correlation with flower yield per plant (0.771) via the indirect positive effect of flower diameter (0.659), vase life (0.353), change in fresh weight at day 3 (0.122), plant height at $90 \mathrm{DAP}(0.012)$ and total chlorophyll content (0.0100.), duration of flowering had a highly significant positive correlation with flower yield per plant (0.800) which was due to the indirect positive effect of duration of crop (5.350), flower diameter (0.296), vase life (0.289), change in fresh weight at day $3(0.138)$, total 
chlorophyll content (0.061)and plant height at 90 DAP (0.0001)Parallel findings were reported by Raghupathi et al., (2019) and Basavaraj (2006) in dahlia; Magar et al., (2010) in gerbera. Hence, direct selection of duration of crop, duration of flowering, flower diameter, vase life, total chlorophyll content and change in fresh weight at day 3 is appropriate for yield improvement.

Table.1 Details of the dahlia genotypes used in present study

\begin{tabular}{|r|l|c|l|}
\hline Sl. No. & \multicolumn{1}{|c|}{ Genotype } & Plant stature & \multicolumn{1}{|c|}{ Flower colour and scheme } \\
\hline $\mathbf{1}$ & Krishna & Tall & Light blend (Pink and light yellow) \\
\hline $\mathbf{2}$ & Barakachri & Tall & Monochromatic (Yellow) \\
\hline $\mathbf{3}$ & Binayananda & Tall & Light blend (orange) \\
\hline $\mathbf{4}$ & Good Day & Tall & Monochromatic (Pink) \\
\hline $\mathbf{5}$ & Glory of India & Tall & Light blend (Tan) \\
\hline $\mathbf{6}$ & M Trangini & Tall & Monochromatic (White) \\
\hline $\mathbf{7}$ & Gargi & Tall & Monochromatic (Red) \\
\hline $\mathbf{8}$ & Master Pic & Tall & Bicolour (Red and white) \\
\hline $\mathbf{9}$ & Hiranmayi & Tall & Monochromatic (Orange) \\
\hline $\mathbf{1 0}$ & Satya Samrat & Tall & Monochromatic (Red) \\
\hline $\mathbf{1 1}$ & Silpa & Tall & Light Blend (Red and white) \\
\hline $\mathbf{1 2}$ & Santashima & Tall & Monochromatic (White) \\
\hline $\mathbf{1 3}$ & Sachin & Tall & Monochromatic (White) \\
\hline $\mathbf{1 4}$ & Pagaltahaker & Tall & Monochromatic (Yellow) \\
\hline $\mathbf{1 5}$ & Eternity & Tall & Bicolour (Red and white) \\
\hline $\mathbf{1 6}$ & Buddha's Mother & Tall & Monochromatic (Orange) \\
\hline $\mathbf{1 7}$ & Santi & Tall & Monochromatic (Red) \\
\hline $\mathbf{1 8}$ & Jayal Singh & Tall & Light blend (White and red) \\
\hline $\mathbf{1 9}$ & Nilkamal & Medium & Monochromatic (Yellow) \\
\hline $\mathbf{2 0}$ & Salini & Medium & Monochromatic (Pink) \\
\hline $\mathbf{2 1}$ & Pusona & Medium & Light Blend (White and maroon) \\
\hline $\mathbf{2 2}$ & Jisu & Medium & Light Blend (White and orange) \\
\hline $\mathbf{2 3}$ & Sowmitha & Medium & Monochromatic (Red) \\
\hline $\mathbf{2 4}$ & Kaviguru & Medium & Monochromatic (Pink) \\
\hline $\mathbf{2 5}$ & WOK & Medium & Monochromatic (Orange) \\
\hline $\mathbf{2 6}$ & Sourav & Dwarf & Monochromatic (Yellow) \\
\hline $\mathbf{2 7}$ & Guddy & Dwarf & Monochromatic (Orange) \\
\hline $\mathbf{2 8}$ & OK & Dwarf & Light Blend (Orange and yellow) \\
\hline $\mathbf{2 9}$ & YBK & Dwarf & Light Blend (White and orange) \\
\hline $\mathbf{3 0}$ & YK & Donochromatic (Orange) \\
\hline $\mathbf{3 1}$ & WBK & Monochromatic (Orange) \\
\hline $\mathbf{3 2}$ & WK & & \\
\hline & & & \\
\hline
\end{tabular}


Table.2 Genotypic and phenotypic correlation co-efficient for growth, flowering, quality and yield parameters in dahlia genotypes

\begin{tabular}{|c|c|c|c|c|c|c|c|c|c|c|c|c|c|c|}
\hline & PH & LAI & PS & DC & DF & TW & FD & SL & VL & WU3 & CF3 & CHL & FPP & \multirow{14}{*}{ II } \\
\hline PH & 1 & -0.121 & $0.727 * *$ & 0.223 & $0.223^{*}$ & -0.019 & $0.836 * *$ & $0.702 * *$ & $0.488 * *$ & $0.638 * *$ & 0.194 & $-0.342 * *$ & -0.021 & \\
\hline LAI & -0.121 & 1 & -0.058 & $0.632 * *$ & $0.632 * *$ & $0.635 * *$ & 0.113 & 0.149 & $0.314^{*}$ & $0.247^{*}$ & $0.397 * *$ & -0.168 & $0.617 * *$ & \\
\hline PS & $0.654 * *$ & -0.061 & 1 & $0.459 * *$ & $0.426 * *$ & 0.154 & $0.689 * *$ & $0.624 * *$ & $0.326^{* *}$ & $0.498 * *$ & -0.046 & -0.015 & 0.216 & \\
\hline DC & 0.206 & $0.585^{* *}$ & $0.337 * *$ & 1 & $0.914 * *$ & $0.807 * *$ & $0.263^{*}$ & $0.344 * *$ & $0.459 * *$ & $0.498 * *$ & $0.291 *$ & 0.016 & $0.771 * *$ & \\
\hline DF & 0.206 & $0.585^{* *}$ & $0.337 * *$ & $0.835 * *$ & 1 & $0.832 * *$ & 0.118 & 0.076 & $0.375^{* *}$ & $0.290 *$ & $0.329 * *$ & 0.103 & $0.800 * *$ & \\
\hline TW & -0.01 & $0.609 * *$ & 0.141 & $0.692 * *$ & $0.798 * *$ & 1 & 0.09 & $0.250 *$ & $0.235^{*}$ & 0.188 & $0.414 * *$ & 0.228 & $0.668 * *$ & \\
\hline FD & $0.746^{* *}$ & 0.102 & $0.575 * *$ & 0.1987 & 0.113 & 0.075 & 1 & $0.722 * *$ & $0.677 * *$ & $0.716^{* *}$ & $0.320 * *$ & $-0.300 *$ & 0.139 & \\
\hline SL & $0.667 * *$ & 0.146 & $0.548 * *$ & $0.315^{*}$ & 0.063 & 0.225 & $0.623 * *$ & 1 & 0.419 & $0.471 * *$ & 0.244 & 0.002 & 0.042 & \\
\hline VL & $0.483^{* *}$ & $0.303^{*}$ & $0.326 * *$ & $0.409 * *$ & $0.371 * *$ & 0.221 & $0.600 * *$ & $0.406 * *$ & 1 & $0.910 * *$ & $0.393 * *$ & $-0.348 * *$ & 0.237 & \\
\hline WU3 & $0.632 * *$ & 0.245 & $0.456 * *$ & $0.462 * *$ & $0.286^{*}$ & 0.185 & $0.655^{* *}$ & $0.448 * *$ & $0.898 * *$ & 1 & $0.322 * *$ & $-0.509 * *$ & 0.173 & \\
\hline CF3 & 0.182 & $0.381^{* *}$ & -0.042 & $0.273^{*}$ & $0.310^{*}$ & $0.361 * *$ & $0.287^{*}$ & 0.223 & $0.375^{* *}$ & 0.31 & 1 & -0.181 & $0.347 * *$ & \\
\hline CHL & $-0.331 * *$ & -0.167 & -0.011 & 0.007 & 0.098 & 0.218 & $-0.262 *$ & 0.01 & $-0.338 * *$ & $-0.498 * *$ & -0.184 & 1 & 0.213 & \\
\hline FPP & -0.018 & $0.592 * *$ & 0.165 & $0.686^{* * *}$ & $0.778 * *$ & $0.646^{* * *}$ & 0.103 & 0.015 & 0.219 & 0.170 & $0.310^{*}$ & 0.198 & 1 & \\
\hline
\end{tabular}

(PH-Plant height at 90 DAP (cm), LAI-Leaf Area Index, PS-Plant spread in E-W (cm), DC-Duration of crop (days), DF-Duration of flowering (days), TW-Tuber weight (g) FD-Flower diameter $(\mathrm{cm})$, SL-Stalk length $(\mathrm{cm})$, VL-Vase life days, WU3-Water uptake at day $3(\mathrm{ml})$, CF3-Change in fresh weight at day $3(\%)$, CHL-Total chlorophyll content, FPP-Number of flowers per plant

* Significant at $\mathrm{P}=0.05 * *$ Significant at $\mathrm{P}=0.01 \mathrm{r}$ value at $5 \%=0.246$ and $1 \%=0.319$ 
Table.3 Estimates of genotypic and phenotypic path coefficient analysis for growth, flowering, quality and yield parameters in dahlia

\begin{tabular}{|c|c|c|c|c|c|c|c|c|c|c|c|c|}
\hline \multicolumn{13}{|c|}{ Genotypic path coefficient analysis } \\
\hline & PH & LAI & PS & DC & DF & TW & FD & SL & VL & WU3 & CF3 & CHL \\
\hline PH & 0.054 & -0.006 & 0.039 & 0.012 & 0.000 & -0.001 & 0.045 & 0.038 & 0.026 & 0.033 & 0.010 & -0.018 \\
\hline LAI & 0.045 & -0.373 & 0.021 & -0.236 & -0.238 & -0.237 & -0.042 & -0.055 & -0.117 & -0.077 & -0.148 & 0.062 \\
\hline PS & -1.210 & 0.097 & -1.664 & -0.763 & -0.363 & -0.256 & -1.147 & -1.038 & -0.543 & -0.700 & 0.076 & 0.027 \\
\hline DC & 1.309 & 3.699 & 2.684 & 5.848 & 5.350 & 4.722 & 1.539 & 2.009 & 2.686 & 2.526 & 1.705 & 0.097 \\
\hline DF & -0.003 & -1.700 & -0.581 & -2.436 & 2.663 & -2.215 & -0.315 & -0.202 & -0.999 & -0.772 & -0.876 & -0.274 \\
\hline TW & 0.029 & -0.958 & -0.232 & -1.218 & -1.255 & -1.508 & -0.136 & -0.377 & -0.355 & -0.096 & -0.625 & -0.344 \\
\hline FD & 2.095 & 0.283 & 1.727 & 0.659 & 0.296 & 0.226 & 2.506 & 1.809 & 1.696 & 1.715 & 0.802 & -0.754 \\
\hline SL & -1.119 & -0.237 & -0.995 & -0.547 & -0.121 & -0.399 & -1.151 & -1.593 & -0.667 & -0.648 & -0.388 & -0.004 \\
\hline VL & 0.376 & 0.241 & 0.251 & 0.353 & 0.289 & 0.181 & 0.521 & 0.322 & 0.770 & 0.714 & 0.303 & -0.268 \\
\hline WU3 & -1.475 & -0.493 & -1.006 & -1.032 & -0.693 & -0.152 & -1.635 & -0.972 & -2.216 & -2.390 & -0.823 & 1.170 \\
\hline CF3 & 0.081 & 0.166 & -0.019 & 0.122 & 0.138 & 0.173 & 0.134 & 0.102 & 0.164 & 0.144 & 0.419 & -0.076 \\
\hline CHL & -0.204 & -0.099 & -0.009 & 0.010 & 0.061 & 0.136 & -0.179 & 0.001 & -0.207 & -0.291 & -0.108 & 0.595 \\
\hline FPP & -0.021 & $0.618 * *$ & 0.216 & $0.771 * *$ & $0.800 * *$ & $0.668 * *$ & 0.139 & 0.042 & 0.237 & 0.157 & $0.347 *$ & 0.213 \\
\hline \multicolumn{13}{|c|}{ Phenotypic path coefficient analysis } \\
\hline & PH & LAI & PS & DC & DF & TW & FD & SL & $\mathbf{V L}$ & WU3 & CF3 & CHL \\
\hline PH & 0.261 & -0.031 & 0.171 & 0.054 & 0.0005 & -0.002 & 0.195 & 0.174 & 0.126 & 0.159 & 0.047 & -0.086 \\
\hline LAI & -0.049 & 0.405 & -0.024 & 0.237 & 0.253 & 0.247 & 0.041 & 0.059 & 0.122 & 0.083 & 0.154 & -0.067 \\
\hline PS & 0.052 & -0.004 & 0.08 & 0.027 & 0.016 & 0.011 & 0.046 & 0.044 & 0.026 & 0.031 & -0.003 & -0.001 \\
\hline DC & 0.054 & 0.154 & 0.088 & 0.263 & 0.22 & 0.182 & 0.052 & 0.083 & 0.107 & 0.104 & 0.072 & 0.001 \\
\hline DF & 0.0005 & 0.191 & 0.064 & 0.255 & 0.305 & 0.244 & 0.034 & 0.019 & 0.113 & 0.087 & 0.095 & 0.03 \\
\hline TW & 0.0006 & -0.036 & -0.008 & -0.041 & -0.048 & -0.06 & -0.004 & -0.013 & -0.013 & -0.003 & -0.021 & -0.013 \\
\hline FD & 0.082 & 0.011 & 0.063 & 0.021 & 0.012 & 0.008 & 0.109 & 0.068 & 0.066 & 0.068 & 0.031 & -0.028 \\
\hline SL & -0.277 & -0.06 & -0.227 & -0.13 & -0.026 & -0.093 & -0.258 & -0.414 & -0.168 & -0.162 & -0.092 & -0.004 \\
\hline VL & -0.027 & -0.017 & -0.018 & -0.023 & -0.021 & -0.012 & -0.034 & -0.023 & -0.057 & -0.052 & -0.021 & 0.019 \\
\hline WU3 & -0.021 & -0.007 & -0.013 & -0.013 & -0.01 & -0.002 & -0.021 & -0.013 & -0.031 & -0.034 & -0.011 & 0.016 \\
\hline CF3 & 0.023 & 0.047 & -0.005 & 0.034 & 0.039 & 0.045 & 0.036 & 0.028 & 0.047 & 0.041 & 0.125 & -0.023 \\
\hline CHL & -0.117 & -0.059 & -0.004 & 0.002 & 0.035 & 0.077 & -0.093 & 0.003 & -0.12 & -0.17 & -0.065 & 0.355 \\
\hline FPP & -0.018 & $0.592 * *$ & 0.165 & $0.686 * *$ & $0.778 * *$ & $0.646^{* *}$ & 0.103 & 0.015 & 0.219 & 0.152 & $0.310^{*}$ & 0.198 \\
\hline
\end{tabular}

(PH-Plant height at 90 DAP (cm), LAI-Leaf Area Index, PS-Plant spread in E-W (cm), DC-Duration of crop (days), DF-Duration of flowering (days), TW-Tuber weight (g)

FD-Flower diameter (cm), SL-Stalk length (cm), VL-Vase life days, WU3-Water uptake at day 3 (ml), CF3-Change in fresh weight at day 3 (\%), CHL-Total chlorophyll content, FPP-Number of flowers per plant.

$*$ Significant at $\mathrm{P}=0.05 *$ Significant at $\mathrm{P}=0.01 \mathrm{r}$ value at $5 \%=0.246$ and $1 \%=0.319$ Residual effect $=0.195$ Bold: Direct effect Above and below diagonal: indirect effect 
At phenotypic level, high direct positive effect was exhibited by LAI (0.405), total chlorophyll content (0.355) and duration of flowering (0.305) while moderate direct positive effect was exhibited by duration of crop (0.263) and plant height at 90 DAP (0.2610).Change in fresh weight at day 3 (0.125) and flower diameter (0.109) showed a low direct positive effect while negative effect was exhibited by stalk length $(-0.414)$, tuber weight $(-0.060)$, vase life $(-0.057)$ and water uptake at day 3 (-0.034).Plant height at 90 DAP had a non-significant negative correlation with flower yield per plant (0.018) due to negative and indirect effect of stalk length (-0.277), total chlorophyll content $(-0.117)$, LAI (-0.049), vase life $(-0.027)$ and water uptake at day $3(-0.021)$ while there was also an indirect positive effect of flower diameter (0.082), duration of crop (0.054), plant spread in E-W (0.052), change in fresh weight at day $3(0.023)$, tuber weight (0.0006) and duration of flowering (0.0005). Duration of crop showed a highly significant positive correlation with flower yield per plant (0.686) via the indirect positive effect of duration of flowering (0.255), LAI (0.237), plant height at 90 DAP (0.054), change in fresh weight at day 3 (0.034), plant spread in E-W (0.027), flower diameter (0.021) and total chlorophyll content (0.002) while there was an indirect negative effect via stalk length (-0.130), tuber weight (-0.041), vase life (-0.023) and water uptake at day $3(-0.013)$.

Similar reports were confirmed by Karuppaiah and Kumar (2010), Bharati et al., (2014), Panwar et al., (2014), Anuja and Jahnavi (2012) in marigold; Kumari et al., (2017) in China aster; Deka and Paswan (2014) in chrysanthemum. Hence, direct selection of duration of crop, duration of flowering, flower diameter, vase life, total chlorophyll content and change in fresh weight at day 3 is appropriate for yield improvement.
In conclusion, since more emphasis must be given to restricted selection based on positive direct effects rather than indirect effects, direct selection of duration of crop, duration of flowering and flower diameteris appropriate for simultaneous progression of more than one trait, especially in a complex character like yield which influenced by many other traits. Direct selection of traits that had high direct positive effect is appropriate for yield improvement. The residual effects appeared to be considerably low (0.195) which indicated that the characters included in this study explained almost all variability towards yield.

\section{References}

Al-Jibourie, H. A., Miller, P.A., Robinson, H.V., 1958, Genotypic and Environmental variance and covariances in a upland cotton cross of interspecific origin. Agron. J., 50: 633536.

Anuja, S. and Jahnavi, K., 2012, Variability, heritability and genetic advance studies in French marigold (Tagetes patula L.). Asian J. Hort., 7(2): 362-364.

Basavaraj, 2006, Genetic variability studies in dahlia. M.Sc. (Hort).Thesis, Univ. Agric. Sci., Dharwad.

Bharati, T. U., Jawaharlal, M., Kannan, M., Manivannan, N. and Raveendran, M., 2014, Correlation and path analysis in African marigold (Tagetes erecta L.). The Bioscan, 9(4): 1673-1676.

Deka, K. K. and Paswan, L., 2014, Correlation and path analysis studies in chrysanthemum. Int. Information System for the Agric. Sci. Technol., 4(2): 221226.

Dewey, D. R. and Lu, K. H., 1959, A correlation and path coefficient analysis of components of crested wheat grass seed production. Agron. J.51: 575 - 581 .

Hanson, C. H., Robinson, H. P. and Comstock, R. E. 1956. Biometrical studies of yield in segregating populations of Korean 
Lespedeza. Agron. J. 48: 268-272.

Islam, B. M. R., Ivy, N. A., Rasul, M. G. and Zakaria, M., 2010, Character association and path analysis of exotic tomato (Solanum lycopersicum L.) genotypes, Bangladesh J.Pl. Breed. Genet., 23(1):13-18, 2010

Islam, M. S. and S. Khan., 1991, Variability and character association in tomato (Lycopersiconesculentum Mill). Bangladesh J. Pl. Breed. Genet. 4(1-2): 49-53.

Johnson, H. W., H. F. Robinson and R. E. Comstock. 1955. Estimation of genetic and environmental variability in soybeans. Agron. J. 47: 314-318.

Karuppaiah, P., and Kumar, P. S., 2010, Correlation and path analysis in African marigold (Tageteserecta L.). Electronic J. Plant Breed., 1(2): 217-220.

Kumari, P., Kumar, R., Rao, T. M., Dhananjaya, M. V. and Bhargav, V., 2017, Genetic variability, character association and path coefficient analysis in China aster [Callistephus chinensis (L.) Nees]. Hort. Flora Res. Spectr., 6(4): 278-282.

Kumari, P., Kumar, R., Rao, T. M., Dhananjaya, M. V. and Bhargav, V., 2017, Genetic variability, character association and path coefficient analysis in China aster [Callistephus chinensis (L.) Nees]. Hort. Flora Res. Spectr., 6(4): 278-282.

Magar, S. D., Warade, S. D., Nalge, N. A. and Nimbalkar, C. A., 2010, Correlation and path analysis studies in gerbera (Gerbera jamesonii). Int. J. Plant Sci., 5(2): 553-555.
Mahajan, R. C., Wadikar, P. B., Pole, S. P. and Dhuppe, M. V., 2011, Variability, correlation and path analysis studies in sorghum. Res. J Agric. Sci. 2(1):101103.

Mathad, G., Hegde, Reddy B. S. and Mulge, R., 2005, Correlation and path coefficient analysis in African (Tageteserecta L.). The Karnataka J. Hort., 1(3): 22-29.

McGiffen, M. E. Jr., Pantone, D. J. and Masiunas, J. B., 1994, Path analysis of tomato yield components in relation to competition with black and eastern black nightshade. J. American Soc. Hort. Sci. 1119(1): 6-11.

Panwar, S., Singh, K. P., Namita, T. Janakiram, T. and Bharadwaj, C., 2014, Character association and path coefficient analysis in African marigold (Tagetes erecta L.). Int. J. Plt. Res., 27(1): 26-32.

Raghupathi, B., Mitra, S. and Saon, B., 2019, Evaluation of genetic variability, correlation and path co-efficient analysis for cut flower attributing traits in medium decorative dahlia (Dahlia variabilisL.) J. Pharmacognosy and Phytochem., 8(1): 465-469.

Rajiv. K, Deka. B. C. and Venugopalan. R., 2012, Genetic variability and trait association studies in gerbera (Gerbera jamesonii) for quantitative traits. Int. J. Agric. Sci., 82(7): 615-619.

Smith, A. W., 1971, In: A Gardener's Dictionary of Plant Names. Cassell and Company Ltd, London, 390.

Wright, S. 1921. Correlation and causation. J. Agric. Res. 26: 557-558.

\section{How to cite this article:}

Maguluri Sree Devi, G. K. Seetharamu, B. C. Patil, C. N. Hanchinamani, Laxman Kukanoor, D. Satish and Sandhyarani Nishani. 2020. Character Association and Path Co-efficient Analysis for Yield Attributing Traits in Dahlia (Dahlia variabilis L.). Int.J.Curr.Microbiol.App.Sci. 9(08): 2944-2950. doi: https://doi.org/10.20546/ijcmas.2020.908.330 